\title{
Visual phase resolution for gray-scale textures
}

\author{
MARTIN HÜBNER, TERRY CAELLI, and INGO RENTSCHLER \\ University of Munich, Munich, Federal Republic of Germany
}

\begin{abstract}
We compared the effects of two different phase-filter operations (quantization and randomization) on the discriminability of gray-scale textures. The resulting discrimination scores were found to be the same, provided the filter operations had been normalized with respect to the mean absolute difference in phase. This enabled us to show that the phase information of gray-scale textures could be compressed to only 16 phase states-a value that was twice as high as previously suggested. We found it difficult to decide whether the observers based their decisions on Fourier phase or on luminance profile cues, which prompted us to further analyze the results in terms of the percent bit modulation of the phase-filtered images. The latter measure of space-domain differences captured human performance equally well; therefore, our results are not supportive of the notion that the visual system encodes spatial phase information per se.
\end{abstract}

The study of the visual processing of arbitrary grayscale images requires a complete description of the image. This description can be given in the image (i.e., space) domain by the two-dimensional intensity distribution. Alternatively, a unique definition of an image can be obtained from the two-dimensional Fourier transform of the intensity distribution (see Caelli, 1981). The Fourier representation can be envisaged as the decomposition of the image into harmonic components, with each spatial frequency component being defined by its amplitude and phase values. The amplitude determines the contrast of each component, and the phase defines its position with respect to the frequency coordinate system. It has been suggested that the Fourier decomposition more closely resembles actual coding properties in primary visual processing stages than do the image domain representations (for reviews, see Julesz \& Schumer, 1981; Pollen \& Ronner, 1983). However, most studies in this area have been concerned with the processing of onedimensional grating patterns with a few frequency components. It is the aim of this report to further investigate the detectability of changes in the frequency spectrum due to phase alterations in suprathreshold two-dimensional images.

Of particular interest in the application of Fourier analysis to image processing are the resolution limits of the visual system to the corresponding spatial frequency information. In two studies, Caelli and Hübner $(1983,1984)$ showed that human observers were largely insensitive to changes in the amplitude information if these changes were restricted to the two-dimensional bandwidths of about $1 / 4$ octave and $10^{\circ}$. Thus, they established a very efficient

This study was supported by Deutsche Forschungsgemeinschaft Re 337/4. T. Caelli participated in this study as Deutsche Forschungsgemeinschaft Guest-Professor (Mu 93/103-1) at the University of Munich. T. Caelli's permanent address is Department of Psychology, University of Alberta, Edmonton, AB T6G 2E9, Canada. Address correspondence to Ingo Rentschler, Institute for Medical Psychology, University of Munich, Goethestr. 31, D-8000 Munich 2, Federal Republic of Germany. code for the processing of image energy. This result is in agreement with the findings of Caelli, Brettel, Rentschler, \& Hilz (1983) concerning increment thresholds of grating spatial frequency and orientation. However, as demonstrated by Oppenheim and Lim (1981) and Brettel, Caelli, Hilz, and Rentschler (1982), it is the phase spectrum that determines the image structure. Consequently, a number of reports have been concerned with observers' sensitivity to phase-related changes in luminance profiles (Badcock, 1984; Burr, 1980; Caelli \& Bevan, 1982; Lawden, 1983; Rentschler \& Treutwein, 1985).

Caelli and Bevan (1982) were the first to determine the resolution limits for two-dimensional phase information in gray-scale images with many frequency components. They investigated the discriminability of original grayscale textures and of phase-quantized versions of these textures as a function of contrast. In a phase-quantized image (see Goodman \& Silvestri, 1970), the phase spectrum contains only a finite number of phase states. For example, a phase-quantization step of $90^{\circ}$ results in only four phase values $\left(0^{\circ}, 90^{\circ}, 180^{\circ}\right.$, and $\left.270^{\circ}\right)$, and the phase of each frequency component is set to the closest of these values without changing its amplitude. When the quantization steps are increased in size, the corresponding distortions in the intensity distribution also increase. Caelli and Bevan determined the contrast threshold for the discrimination of the original and of its phasequantized versions. They found that images quantized in $15^{\circ}$ steps could not be discriminated from the originals.

Because phase quantization restricts the spectrum to a few phase values and brings about distortions of the image structure (see Figure 1), it is not clear whether these distortions are due only to the loss of phase information or whether the relative enhancement of certain phase states is also important. Therefore, the phase-quantization technique as such does not allow for direct conclusions as to the redundancy in phase information. For this reason, we decided to compare the results of phase quantization with 

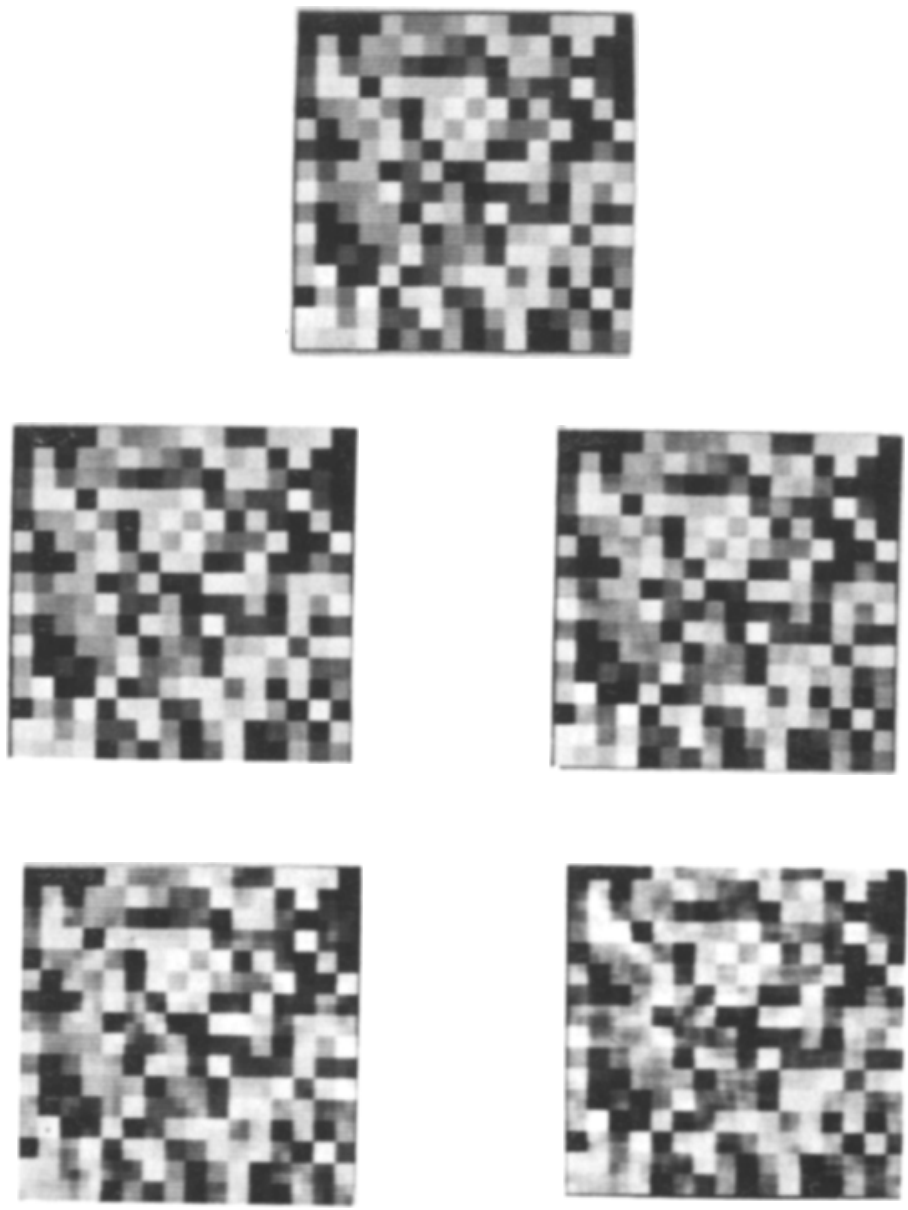

Figure 1. Top: Original $128 \times 128$ six-bit pixel texture. Second row: Phasequantized texture (left) and phase-randomized texture (right) with a phase resolution of $20^{\circ}$. Third row: Sanee as in second row but with a phase resolution of $45^{\circ}$.

those of an alternative phase-distorting filter operation. The latter procedure also reduces the phase information content of an inage, but it does not impose restrictions on the distribution of phase values within the phase domain. We will refer to this process as phase randomization because it replaces the phase of each spatial frequency component of an image by a new value that is generated by means of a random process (for details on this procedure, see the Method section).

Another problem with the investigation of the perceptual consequences of image-phase modulation is that phase coding per se and the detection of phase-related changes in image luminance profiles must not be confounded (Badcock, 1984; Rentschler \& Treutwein, 1985). This prompted us to use an image domain measure as well; that is, we used the average absolute pixel difference (or percent bit modulation) to compare the usefulness of space and spectral domain representations for the characterization of the effects of image-phase modulation.

\section{METHOD}

\section{Subjects}

Two observers (aged 29 and 30) with normal or corrected-tonormal visual acuity, one of whom was the first author, participated in the experiments.

\section{Stimuli}

The original image used throughout the study was a random checkerboard texture of $128 \times 128$ pixels with a check size of $8 \times 8$ pixels. The gray levels spanned a full six-bit linear range (64 gray values). This image was Fourier transformed by the Cooley-Tukey Fast Fourier Transform (FFT), and the phase spectrum was quantized or randomized with steps of $10^{\circ}, 15^{\circ}, 20^{\circ}, 24^{\circ}, 30^{\circ}, 36^{\circ}$, $40^{\circ}$, and $45^{\circ}$.

The process used to generate the phase-quantized versions of the original gray-scale texture was the same as that used by Caelli and Bevan (1982). With phase randomization, corresponding steps define the lower and upper bounds for the random numbers. For example, a randomization step of $90^{\circ}$ means that an original phase value of, say, $30^{\circ}$ is replaced by a random phase angle that must lie in the range of $0^{\circ}$ to $90^{\circ}$. An original phase value of $295^{\circ}$ is 
replaced by a number that lies between $270^{\circ}$ and $360^{\circ}$, and so forth. The subsequent inverse Fourier transform resulted in 16 phasedistorted images, examples of which are shown in Figure 1.

In a previous study (Caelli, Hübner, \& Rentschler, 1985), we found that observers were about equally sensitive to phase changes in different frequency bands. Therefore, in the present experiment, the phase alterations were enacted over the full frequency spectrum. It is also important to note that these frequency domain filter processes do not change the amplitudes of the spatial frequency components. Consequently, all images had an identical space average luminance $\left(60 \mathrm{~cd} / \mathrm{m}^{2}\right)$ and a peak contrast value of $60 \%\left[\left(1_{\max }-\right.\right.$ $\left.1_{\min }\right) /\left(1_{\max }+1_{\min }\right), 1=$ luminance]. Note also that the original texture's phase spectrum had a uniform density. The randomization process, by definition, does not destroy this property, whereas the quantization process, as used previously by Caelli and Bevan (1982), results in only a few discrete phase states.

The images were displayed by a Videograph image-processing system (interfaced with a DEC LSI 11/23 laboratory computer) on a BG 123 Grundig television monitor with P32 phosphor. The images subtended a visual angle of $2^{\circ}$ at a viewing distance of $1.7 \mathrm{~m}$.

\section{Procedure}

Because we were concerned with the precise determination of the phase resolution threshold for suprathreshold images, we did not use Caelli and Bevan's (1982) contrast paradigm. Instead, we established psychometric functions, with the phase distortion steps being the independent variable.

The trials were designed to provide discrimination sensitivities between the original image and its phase-distorted versions. Each trial consisted of an initial presentation of the original texture for $200 \mathrm{msec}$, followed by one of the phase-distorted images in the same spatial aperture for $200 \mathrm{msec}$, with an interstimulus interval of $700 \mathrm{msec}$. Between the trials and during the interstimulus period, the luminance of the screen was set to the space average luminance of the targets. The observers were instructed to respond as to whether they believed the two images shown were identical or different, using confidence ratings between 1 and 3 for same and 4 to 6 for different. One run consisted of 20 replications for four distorted images $\left(10^{\circ}, 20^{\circ}, 30^{\circ}\right.$, and $40^{\circ}$ or $15^{\circ}, 24^{\circ}, 36^{\circ}$, and $\left.45^{\circ}\right)$ from each of the two filter processes (quantization or randomization) and 40 catch trials (original texture followed by original). The second (probe) image types were presented in random order. The subjects initiated each trial by pressing a button. Each run was repeated three times, which resulted in $240 \times 3 \times 2=1,440$ trials for each observer.

\section{Data Analysis}

The sensitivity scores were obtained by calculating the area $P(A)$ under the receiver operating characteristic (ROC). A maximumlikelihood method for estimating the parameters of the signal detection model was used. This method was developed by Dorfman and Alf (1969), and a more recent version of the related program RSCORE was provided by Harvey (1984). Note that a value of $P(A)$ $=0.5$ corresponds to guessing, and a value of 1.0 corresponds to $100 \%$ correct. It is also of interest that $P(A)$-the area under the ROC curve-may be interpreted as the probability of a correct response in a two-alternative forced choice situation (Green \& Swets, 1974).

\section{RESULTS AND DISCUSSION}

The results for the 2 observers are shown in Figure 2. As expected, the discrimination scores increased with increasing phase distortion steps, but scores to randomization (solid lines) clearly exceeded those for phase quantization (dashed lines) with respect to the step size. This came as a surprise because, for each step, the filter operations were enacted over the same phase range.

To further analyze the results, logistic functions of the form

$$
f(x)=\frac{\exp \left(p_{1}+p_{2} x\right)}{1+\exp \left(p_{1}+p_{2} x\right)}
$$

were fitted to the data (computer program PLR, BMDP statistical software, Dixon, 1981). Here, we assumed that at zero phase shift (identical patterns), the observers would behave in an unbiased manner, which would result in $P(A)$ $=0.5$. Since this was evidenced from our results, we used this offset value in fitting the logistic function. We then calculated the differences at $P(A)=0.75$ between the two

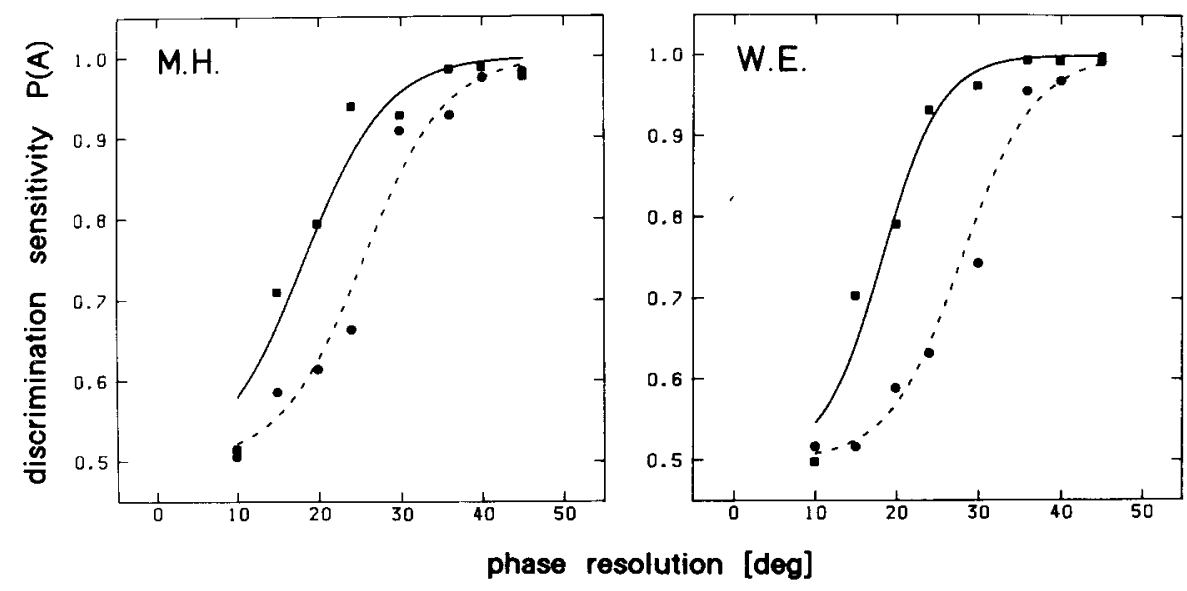

Figure 2. Discrimination sensitivities $[P(A)=$ area under the ROC curve] for phase-quantized textures (circles, dashed lines) and phase-randomized textures (squares, solid lines) as a function of phase resolution. Logistic functions were fitted to the data points (see text). 
curves in order to obtain some measure for the difference in discrimination scores to phase quantization and randomization. The results were $7.1^{\circ}$ (M.H.) and $9.7^{\circ}$ (W.E.). These differences do not necessarily imply that the phase randomization process is, per se, a more perceptually salient method of destroying image structure. This is because the mean (or total) absolute differences in phase between the original texture and its phasemodulated versions were not identical for both methods.

\section{A Phase Domain Measure of Image Distortion}

To obtain a measure for those differences that were independent of the specific type of phase modulation employed, we defined the mean absolute difference in phase (MAD) between the original $(o)$ and filtered $(f)$ versions thereof as:

$$
\operatorname{MAD}=\frac{1}{n^{2}} \sum_{k} \sum_{m}\left|\phi_{o}(k, m)-\phi_{f}(k, m)\right|
$$

When the discrimination scores shown in Figure 2 are replotted as functions of the mean absolute phase differences between the original and the phase-altered spectra, the apparent differences between the two processes effectively disappear (Figure 3). In other words, the differences in discrimination sensitivities documented in Figure 2 do not reflect differences in sensory processing, but rather must be attributed to differences in the amount of physical image distortions as a result of the respective phase modulations.

In summary, these results show that, regardless of the type of phase modulation employed, observers cannot detect changes in the structure of gray-scale textures if the mean absolute deviation from the original spectrum is below about $5^{\circ}$. Moreover, the results of both phase manipulations are equally detectable above threshold; that is, identical discrimination scores are produced if the mean absolute difference is considered.

\section{Image Information Compression}

It would thus seem that, due to the apparent equality in image distortion found here, the quantization procedure is as adequate as any other encoding technique for phase information compression. Figure 2 shows that the threshold, in phase step size, is about $25^{\circ}$, whereas in Figure 4, we show that this resolution limit seems to hold also for more "natural" images than textures. In the top row, two original images $(256 \times 256$ pixels, 64 gray values) are shown. These images were phase quantized (second row) to only 16 phase values $\left(360 / 16=22.5^{\circ}\right.$ quantization step), and even careful inspection reveals almost no differences when compared with the originals. Therefore, it can be concluded that 16 possible phase states for each frequency component are sufficient to encode the phase spectrum of such images. Together with the briefly mentioned efficient amplitude code (Caelli \& Hübner, 1984), these findings might also have implications for certain applications in digital image processing, and, indeed, issues of "efficient" detector profiles for biological image processing (see Pollen, Foster, \& Gaska, 1985).

\section{Measuring Image Distortion in the Intensity Domain}

In our experiments and demonstrations, we were concerned with the detectability of changes in the image structure of two-dimensional gray-scale textures brought about by precisely defined filter operations of image phase spectra. By definition, these filter operations allow for the investigation of the discriminability of images with identical power spectra and total energies. This type of experiment, however, does not allow for conclusions concerning the underlying mechanisms that are responsible for the discrimination behavior. In fact, Rentschler and Treutwein (1985) showed that in the case of onedimensional grating patterns with two frequency components, the phase difference between the first and third har-

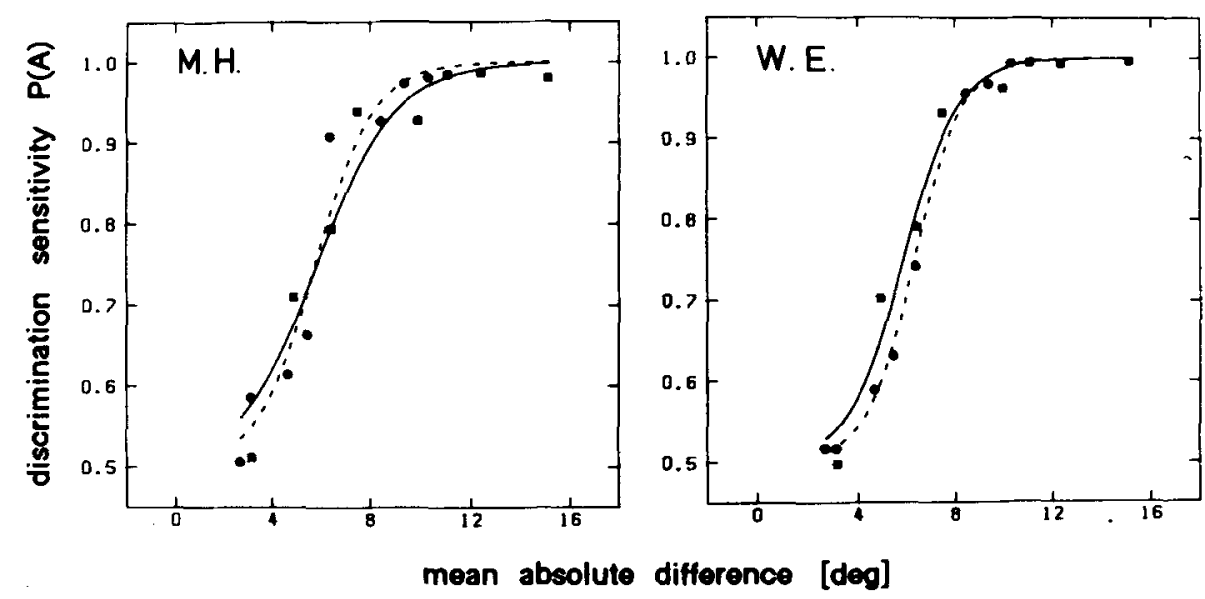

Figure 3. Discrimination sensitivities $[P(A)]$ for phase-quantized textures (circles, dashed lines) and phase-randomized textures (squares, solid lines) as a function of the mean absolute difference in phase. 

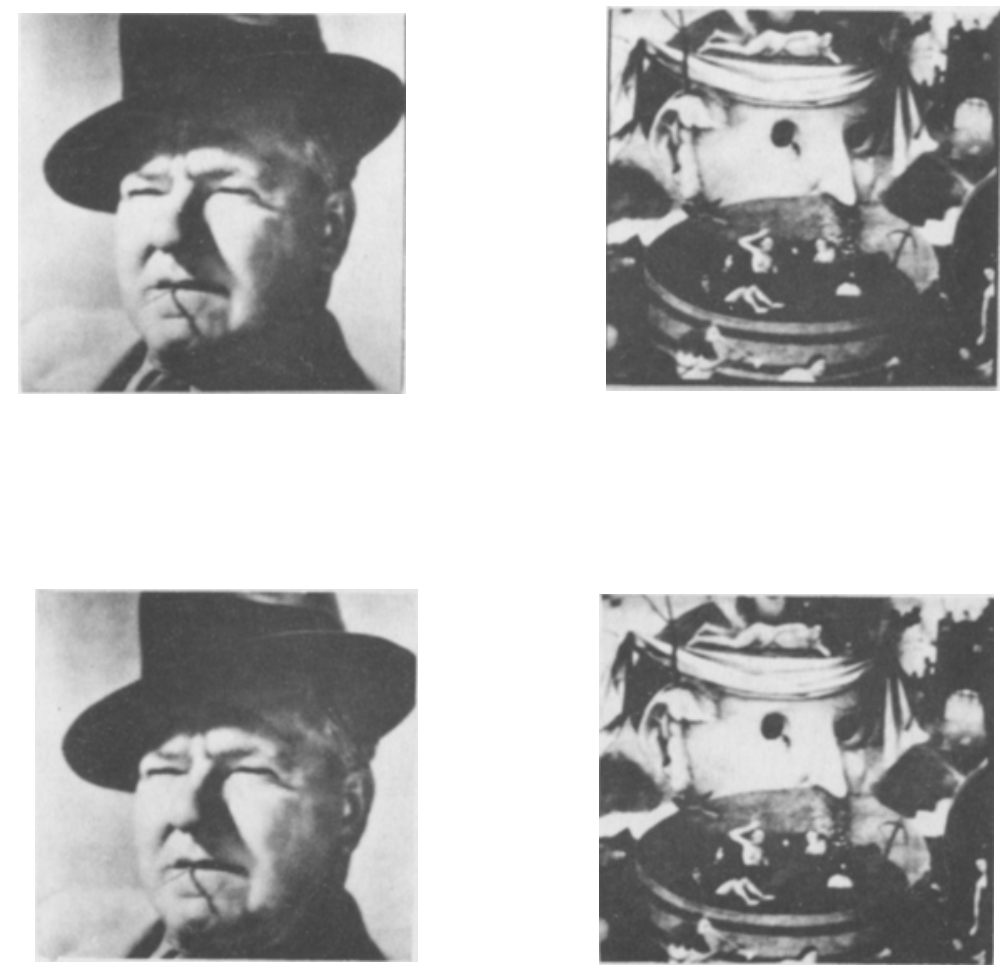

Figure 4. Top row: Two original images ( $256 \times 256$ pixels) with 64 gray values. Second row: Phase-quantized versions with a phase resolution of $22.5^{\circ}$ (16 phase values).

monic did not predict human discrimination performance when foveal and extrafoveal vision was compared. They concluded that their results were incompatible with phase coding per se (see also Badcock, 1984; Watt, 1985), and that local measures of the intensity profiles had to be taken into account. Therefore, one would expect that some intensity-domain measure for the difference between the original and the phase-distorted images should predict equally well discrimination performance. Hence, we have also calculated the average absolute pixel difference (percent bit modulation, or PBM) between the original texture and the phase-filtered images. This intensity domain measure of image distortion is defined by:

$$
\mathrm{PBM}=100 \cdot \frac{1}{n^{2}} \sum_{k} \sum_{m} \frac{\left|\mathrm{I}_{o}(k, m)-\mathrm{I}_{f}(k, m)\right|}{\mathrm{I}_{o}(k, m)},
$$

in which $o=$ original and $f=$ filtered images.

In Figure 5, the discrimination scores obtained from our experiment are replotted against the corresponding PBM. It can be seen that this formula predicts discrimination performance very well. We therefore conclude that, at least within the present experiments, image domain (pixel differences) and Fourier domain (phase differences) measures both predict equally well the ability to discriminate two images that differ due to precisely defined phase-filter operations. In this sense, the image-domain and spectrum-domain descriptions are envisaged as alter- native stimulus descriptions that are both in accord with human visual performance.

\section{CONCLUSIONS}

We have demonstrated the perceptual equivalence of two types of image-phase modulation-namely, phase quantization and phase randomization-under the condition that the effect of these filter operations was normalized with respect to the mean absolute difference in phase between the original image and its phase-altered versions. Insofar as the issue of the neural encoding of phase information is concerned, this result is hardly compatible with the idea that there are a small number of "phase sensitive devices" being selectively tuned to a few phase states, such as, for instance, $0^{\circ}$ and $180^{\circ}$ (see Atkinson \& Campbell, 1974). It should be kept in mind, however, that our results were obtained by the use of gray-scale textures that were characterized by a uniform distribution of phase states. Hence, it remains to be seen whether the above-mentioned equivalence also holds for other types of images that have uneven distributions of phase states with possible maxima of their density around, say, $0^{\circ}$ or $180^{\circ}$.

The normalization of phase-filter operations in terms of the mean difference in phase between an original image and a phase-altered version of that image enabled us 


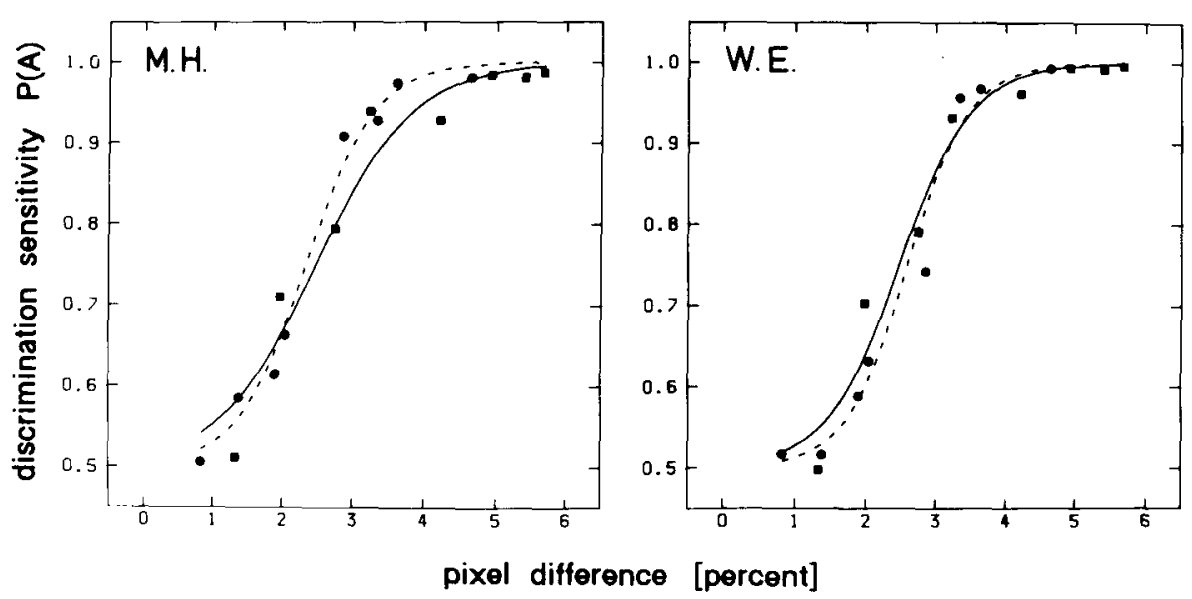

Figure 5. Discrimination sensitivities $[P(A)]$ for phase-quantized textures (circles, dashed lines) and phase-randomized textures (squares, solid lines) as a function of the pixel difference between the original and the phase-filtered images.

to unequivocally answer the question of phase-information compression. We found a minimum of 16 resolvable phase values; that is, we established that an original gray-scale texture could not be discriminated from its phasemodulated version when at least 16 equally distributed phase states were present. This is twice the number previously suggested by Caelli and Bevan (1982). It is also interesting to note that the present value of $22.5^{\circ}$ phase resolution compares well with the value of $30^{\circ}$ obtained by Burr (1980) for the discrimination of compound gratings, and with a detection limit of about $20^{\circ}-30^{\circ}$ as reported by Caelli et al. (1985) for the detectability of band-limited phase shifts in gray-scale textures.

The second type of equivalence that we found was that between phase (or spatial-frequency) domain and intensity (or space) domain measures of image distortion. This is hardly surprising from the mathematical point of view, because the two-dimensional function of luminances across the image (or space) domain is uniquely related to its Fourier integral and, therefore, to its amplitude and power spectra (uniqueness theorem of Fourier transforms; see Korn \& Korn, 1968, p. 140). Yet, it has been proposed that the encoding of images in the brain is more adequately described by spectral image properties (see the introduction). Such a possibility would not necessarily be in conflict with the uniqueness theorem of the Fourier transform, because the implicit assumption of the "Fourier theory of vision"' is that sensory processing is restricted to certain components of the image spectra, thus removing the unique relationship between the physical image and its neural representation. Our present findings are not suggestive of such a notion; however, as with most past experiments, it is difficult to arrive at more precise conclusions as to whether our observers were responding to space or spatial-frequency domain information. Rentschler and Treutwein (1985) were able to solve this problem for the case of compound gratings by using pairs of target images that differed only in the positional relationships of gray levels (iso-second-order joint-probability density, in the case of binary images). Yet it is not clear how such a restriction could be imposed on gray-scale textures, which were the concern of the present study.

\section{REFERENCES}

Atkinson, J., \& Campbell, F. W. (1974). The effect of phase on the perception of compound gratings. Vision Research, 14, 159-162.

BADCOCK, D. R. (1984). Spatial phase or luminance profile discrimination? Vision Research, 24, 613-623.

Brettel, H., Caelli, T., Hilz, R., \& Rentschler, I. (1982). Modelling perceptual distortion: Amplitude and phase processing in the human visual system. Human Neurobiology, 1, 61-67.

Burr, D. C. (1980). Sensitivity to spatial phase. Vision Research, 20, 391-396.

CaEll, T. (1981). Visual perception: Theory and practice. Oxford: Pergamon Press.

CaElli, T., \& BEVAN, P. (1982). Visual sensitivity to two-dimensional phase. Journal of the Optical Society of America, 72, 1375-1381.

Caelli, T., Brettel, H, , Rentschler, I., \& Hilz, R. (1983). Discrimination thresholds in the two-dimensional spatial frequency domain. Vision Research, 23, 129-133.

CaElu, T., \& HüBNER, M. (1983). Coding images in the frequency domain: Filter design and energy processing characteristics of the human visual system. IEEE Transactions on Systems, Man, \& Cybernetics, SMC-13, 1018-1021.

Caelli, T., \& Hübner, M. (1984). On the efficient two-dimensional energy coding characteristics of spatial vision. Vision Research, 23 , 1053-1055.

Caelli, T., Hübner, M., \&entschler, I. (1985). The detection of phase-shifts in two-dimensional images. Perception \& Psychophysics, 37, 536-542.

Dixon, W. J. (Ed.). (1981). BMDP statistical software [Computer program]. Los Angeles: University of California Press.

Dorfman, D. D., \& AlF, E. (1969). Maximum-likelihood estimation of parameters of signal-detection theory and determination of confidence intervals-rating method data. Journal of Mathematical Psychology, 6, 487-496.

Goodman, J. W., \& Silvestri, A. M. (1970). Some effects of Fourierdomain phase quantization. IBM Journal of Research \& Development, 14, 478-484. 
GREeN, D. M., \& Swers, J. A. (1974). Signal detection theory and psychophysics. Huntington, NY: Krieger.

HARVEY, L. O., JR. (1984). Estimation of signal detection parameters: RSCORE user's manual [Computer program manual]. Boulder, $\mathrm{CO}$ : University of Colorado, Department of Psychology.

Julesz, B., \& Schumer, R. (1981). Early visual perception. Annual Reviews of Psychology, 32, 575-627.

KorN, G. A., \& KoRN, T. M. (1968). Mathematical handbook for scientists and engineers (2nd ed.). New York: McGraw-Hill.

LAWDEN, M. C. (1983). An investigation of the ability of the human visual system to encode spatial phase relationships. Vision Research, 23, 1451-1463.

OPPENHEIM, A., \& LiM, J. (1981). The importance of phase in signals. Proceedings of the IEEE, 69, 529-541.
Polien, D. A., Foster, K. M., \& Gaska, J. P. (1985). Phase-dependent responses of visual cortical neurons. In D. Rose \& V. Dobson (Eds.). Models of the visual conex (pp. 282-291). Chichester, England: Wiley.

Pollen, D. A., \& Ronner, S. F. (1983). Visual cortical neurons as localized spatial frequency filters. IEEE Transactions on Sytems, Man, \& Cybernetics, SMC-13, 907-916.

Rentschler, I., \& Treutwein, B. (1985). Loss of spatial phase relationships in extrafoveal vision. Nature, 313, 308-310.

WatT, R. J. (1985). Structured representation in low-level vision. $\mathrm{Na}$ ture, 313, 266-267.

(Manuscript received October 29, 1986; revision accepted for publication October 6,1987 .) 